\title{
Wavelets optimization method for evaluation of fractional partial differential equations: an application to financial modelling
}

\author{
Asmat Ara', Najeeb Alam Khan²*, Oyoon Abdul Razzaq ${ }^{3}$, Tooba Hameed² and \\ Muhammad Asif Zahoor Raja ${ }^{4}$
}

\author{
"Correspondence: \\ njbalam@yahoo.com \\ 2 Department of Mathematics, \\ University of Karachi, Karachi, 75270, \\ Pakistan \\ Full list of author information is \\ available at the end of the article
}

\begin{abstract}
In the present paper, we employ a wavelets optimization method is employed for the elucidations of fractional partial differential equations of pricing European option accompanied by a Lévy model. We apply the Legendre wavelets optimization method (LWOM) to optimize the governing problem. The novelty of the proposed method is the inclusion of differential evolution algorithm (DE) in the Legendre wavelets method for the optimized approximations of the unknown terms of the Legendre wavelets. Sequentially, the functions and components of the pricing models are discretized by utilizing the operational matrix of fractional integration of Legendre wavelets. Illustratively, the implementation of the LWOM is exemplified on a pricing European option Lévy model and successfully depicted the stock paths. Moreover, comparison analysis of the Black-Scholes model with a class of Lévy model and LWOM with q-homotopy analysis transform method (q-HATM) is also deliberated out. Accordingly, the technique is found to be appropriate for financial models that can be expressed as partial differential equations of integer and fractional orders, subjected to initial or boundary conditions.
\end{abstract}

Keywords: pricing models; Lévy processes; wavelets approximation; optimization

\section{Introduction}

The study of financial theory is a versatile field that connects the assumptions of finance and techniques of mathematics. With the expeditious expansion of financial derivatives like options and futures, it has incited the attention of researchers particularly toward the area of pricing models. In 1973, Black, Scholes, and Merton made an innovative assumption that the stock price and other observable quantities that depend on the volatility of the option price are explicitly related to hedging approaches. The considerable inspiration of the Black-Scholes-Merton (BS) model defines that by keeping a certain quantity of stock the risk-free rate of the option price, known as delta, is a dynamically hedged option situation. On this key innovation, Scholes and Merton were awarded the Nobel prize in 1997. After the development of the BS model, many endeavors have been organized to sort out the rigorous notions of pricing models. Merton [1] proposed a Merton jump diffusion

(c) The Author(s) 2018. This article is distributed under the terms of the Creative Commons Attribution 4.0 International License (http://creativecommons.org/licenses/by/4.0/), which permits unrestricted use, distribution, and reproduction in any medium, provided you give appropriate credit to the original author(s) and the source, provide a link to the Creative Commons license, and indicate if changes were made. 
model (MJD) by considering a symmetric $\alpha$-stable Lévy process in place of an exponential Brownian motion. Many advancements have been contrived on the MJD model by considering the Lévy models with jumps. Bates [2] discovered that the stochastic volatility and elucidated the 'volatility smile'. Under the systematic jumps and volatility risk, the Heston model [3] merged a stochastic volatility and a jump-diffusion process (SVJD). Some stochastic models with infinite activity pure jump processes, such as FMLS (finite moment log stable), CGMY (Carr-Madan-Geman-Yor) and KoBol (Koponen-BoyarchenkoLevendorskii) can be found in [4-12]. The characteristics of these price models are more flexible and present realistic descriptions of the price process at various time scales.

Fractional derivatives offer a more delicate mechanism for many fields, in comparison with the integer-order derivatives, to confine the characteristics of processes, materials, etc. This special branch has analyzed various problems of different aspects by many researchers and scientists. Numerous papers have been published in this regard to explore and enhance the definitions and properties in order to overcome the inadequacies of previous definitions of fractional calculus, such as conformable derivatives [13], fractional derivatives with smooth [14], nonlocal and nonsingular kernel [15], etc. [16, 17]. Many researchers have proposed different techniques to solve fractional partial differential equations numerically and analytically. A general finite difference scheme is applied on three FPDEs under some infinite activity Lévy models (FMLS, KoBol, and CGMY) using the Grunwald-Letnikov definition [18]. The approach in [19] is based on a combination of the Laplace transformation and homotopy methods for the approximate analytical solution of FPDEs in the Liouville-Caputo and Caputo-Fabrizio sense. In [20] a new Adomian decomposition method based on conformable derivatives is utilized to solve FPDEs. The local fractional derivative with homotopy perturbation Sumudu transform technique is studied to solve FDEs in [21]. Hence, by means of different theories of fractional derivatives, the behaviors of many FPDEs have been studied, and various techniques have been developed $[22,23]$.

Motivated by the worth-mentioning research works found in the literature, in this endeavor, exploring the applications of FPDEs in financial mathematics, we consider the BS model and a class of Lévy models (FMLS), which is used to model stock price $[5,18]$. The proposed method, the Legendre wavelets optimization method (LWOM), is successfully implemented on BS and FMLS. The technique is an amalgamation of the Legendre wavelets approximation and differential evolution algorithm. The Legendre wavelets method has been extensively used to approximate the unknown functions of integer- and fractional-order differential equations $[24,25]$. Besides, the differential evolution (DE) algorithm, the famous meta-heuristic scheme, has nowadays gained popularity for its global optimization attribute $[26,27]$. Here, after discretizing the functions using the Legendre wavelets, the equations are optimized using the DE scheme to find the unknown parameters. The transparent numerical comparison of the BS and FMLS models with q-homotopy analysis transform method (q-HATM) [18] also expounded.

The outline of the paper is as follows. In Section 2, the fractional definition and properties are outlined. In Section 3, financial and mathematical backgrounds of pricing options models are acquainted. Key features of LWOM are explained in Section 4, whereas the implementation of the method on European call option model and discussion of results is given in Section 5. Section 6 contains the conclusive annotations observed from the facts and figures of the whole study. 


\section{Fractional prerequisites}

In this section, we give a few basic results and definitions from fractional calculus, which are helpful for the advance evolution.

Definition 2.1 Let $v>0, m=\lceil\nu\rceil$, and $\phi(z, \tau) \in C^{m}\left(\Re \times \Re_{+}\right)$. Then the Caputo fractional derivative of $\phi(z, \tau)$ with respect to $\tau$ is defined as $D_{\tau}^{v} \phi(z, \tau)=\mathrm{I}_{\tau}^{m-v} \frac{\partial^{m}}{\partial \tau^{m}} \phi(z, \tau)$, where $\mathrm{I}_{\tau}^{m-\nu}$ is the Riemann-Liouville fractional integral given as

$$
\mathrm{I}_{\tau}^{m-v} \phi(z, \tau)=\frac{1}{\Gamma(v)} \int_{0}^{\tau}(\tau-s)^{\nu-1} \phi(z, s) d s .
$$

Some essential properties of fractional differential and integral operators are are the following:

(i) $\mathrm{I}_{\tau}^{\nu} \mathrm{I}_{\tau}^{\mu} \phi(z, \tau)=\mathrm{I}_{\tau}^{\nu+\mu} \phi(z, \tau)=\mathrm{I}_{\tau}^{\mu} \mathrm{I}_{\tau}^{v} \phi(z, \tau)$.

(ii) $\mathrm{D}_{\tau}^{v} \mathrm{I}_{\tau}^{\mu} \phi(z, \tau)=\mathrm{I}_{\tau}^{\mu-v} \phi(z, \tau)$.

(iii) $I_{\tau}^{v} \mathrm{D}^{\mu} \phi(z, \tau)=\phi(z, \tau)-\sum_{k=0}^{n-1} \frac{\tau^{k}}{k !} \frac{\left.\partial^{k} \phi(z, \tau)\right|_{\tau=0}}{\partial \tau^{k}}$.

(iv) The Caputo fractional derivative of order $v>0$ for $q(\tau)=\tau^{\alpha}$ is

$$
\mathrm{D}_{\tau}^{v} q(\tau)= \begin{cases}\frac{\Gamma(\alpha+1)}{\Gamma(\alpha-\nu+1)} \tau^{\alpha-\alpha} & \text { if } m>v \geq m-1, \\ 0 & \text { if } v \in\{0,1,2, \ldots, m-1\} .\end{cases}
$$

For more details, see [28].

Lemma 2.2 Let $m-1<v \leq m$ and $\phi(\cdot, \tau) \in C^{m}([0, T])$. Then $\mathrm{I}_{\tau}^{v} \mathrm{D}_{\tau}^{\mu} \phi(z, \tau)=\phi(z, \tau)-$ $\sum_{i=0}^{m-1} \eta_{i}(z) \tau^{i}$, where $\eta_{i}(z)=\frac{1}{i !} \frac{\partial^{i} \phi(z, \tau)}{\partial \tau^{i}}$.

Definition 2.3 The two-parameter Mittag-Leffler function $E_{\nu, \mu}(\tau)$ is defined as

$$
E_{v, \mu}(\tau)=\sum_{\kappa=0}^{\infty} \frac{\tau^{\kappa}}{\Gamma(\nu \kappa+\mu)}, \quad \tau \in \Re, \nu, \mu>0
$$

Definition 2.4 The left-handed and the right-handed Riemann-Liouville fractional derivatives [28] of order $v$ are defined as

$$
\begin{aligned}
& \left(\mathrm{D}_{\mathrm{L}+}^{v} \phi\right)(\tau)=\frac{d^{v} \phi(\tau)}{d_{+} \tau^{v}}=\frac{1}{\Gamma(m-v)} \frac{d^{m}}{d_{+} \tau^{m}} \int_{\mathrm{L}}^{\tau} \frac{\phi(\eta)}{(\tau-\eta)} d \eta, \\
& \left(\mathrm{D}_{\mathrm{R}-}^{v} \phi\right)(\tau)=\frac{d^{v} \phi(\tau)}{d_{-} \tau^{v}}=\frac{1}{\Gamma(m-v)} \frac{d^{m}}{d_{-} \tau^{m}} \int_{\tau}^{\mathrm{R}} \frac{\phi(\eta)}{(\eta-\tau)} d \eta,
\end{aligned}
$$

where $m$ is an integer such that $m-1<v \leq m$. Equations (2) and (3) satisfy the standard integer derivatives if $v=m$, that is,

$$
\left(\mathrm{D}_{\mathrm{L}+}^{m} \phi\right)(\tau)=\frac{d^{m} \phi(\tau)}{d \tau^{m}}
$$

and

$$
\left(\mathrm{D}_{\mathrm{R}-}^{m} \phi\right)(\tau)=(-1)^{m} \frac{d^{m} \phi(\tau)}{d \tau^{m}}=\frac{d^{m} \phi(\tau)}{d(-\tau)^{m}} .
$$




\section{Background of option pricing model}

The mathematical and financial background of pricing options is described here in order to identify the problem. The Brownian motion is a famous stochastic process and is a part of the Black-Scholes model. It is profoundly recognized as a Wiener process, named after Norbert Wiener, who was the first to explain the process mathematically [29].

The model proposed by Black and Scholes is an approximation of the underlying asset as a geometric Brownian motion (GBM) [6]. In a market, the BS model has two different assets, a risk-free asset $B$ and a risky asset $S$, mathematically defined by

$$
\begin{aligned}
& d B_{t}=r B_{t} d t, \\
& d S_{t}=r S_{t} d t+\sigma S_{t} d \mathrm{~W}_{t},
\end{aligned}
$$

where $r$ is the interest rate, $\sigma$ is the volatility, and W is the Wiener process.

The BS PDE for a European option can be modeled as [18]

$$
\begin{aligned}
& \frac{\partial v(S, t)}{\partial t}+\frac{1}{2} \sigma^{2} S^{2} \frac{\partial^{2} v(S, t)}{\partial S^{2}}+r S \frac{\partial v(S, t)}{\partial S}-r(S, t)=0, \\
& v(S, T)=\phi(S) .
\end{aligned}
$$

The more standard type of stochastic process is a Lévy process in which the Brownian motion is an example. It consists of three terms, drift, diffusion, and a jump, which play a crucial role in constructing different market models.

In financial mathematics, pricing derivatives are studied by means of FPDEs. Here, we investigate the following Lévy model in the form of fractional diffusion equations, named as finite moment log stable model (FMLS)

$$
\frac{\partial v(x, t)}{\partial t}+A \frac{\partial v(x, t)}{\partial x}+B(x) \frac{\partial^{\gamma}(f(x) v(x, t))}{\partial_{+} x^{\gamma}}+C v(x, t)=0
$$

subject to the conditions

$$
v(x, T)=s(x), \quad v(L, t)=0, \quad v(R, t)=b(R, t)
$$

for $1 \leq \gamma \leq 2, L \leq x \leq R$, and $0 \leq t \leq T$. The right-handed Riemann-Liouville fractional derivative, defined in Eq. (2), is used to approximate the spatial $\gamma$ th-order fractional derivative.

The structure of FMLS model under the European call option is given by Eq. (9) with

$$
A=\left(r+\frac{1}{2} \sigma^{\gamma} \sec \left(\frac{\gamma \pi}{2}\right)\right), \quad B(x)=\left(-\frac{1}{2} \sigma^{\gamma} \sec \left(\frac{\gamma \pi}{2}\right)\right), \quad f(x)=1, C=r,
$$

and the conditions

$$
v(x, T)=\max \left(e^{x}-K, 0\right), \quad v(L, t)=0, \quad \text { and } \quad v(R, t)=\max \left(e^{R}-K e^{-r(T-t)}, 0\right) .
$$

The approximations of the BS and FMLS models are carried out by using the LWOM, described in the next section. 


\section{Features of Legendre wavelets optimization method}

\subsection{The Legendre wavelets approximation}

On continuous variation of dilation parameter $\alpha$ and translation parameter $\beta$, the family of continuous wavelets is retrieved, that is,

$$
\eta_{\alpha, \beta}(\xi)=|\alpha|^{-k} \eta\left(\frac{\xi-\beta}{\alpha}\right), \quad \alpha, \beta \in \mathbf{R}, \alpha \neq 0
$$

If parameters $\alpha$ and $\beta$ are restricted to $\alpha=2^{-k}$ and $\beta=\hat{n} 2^{-k}$, where $\hat{n}=2 n-1$, with $n=$ $1,2, \ldots, 2^{k-1}$ and $k$ is any positive integer, then the following family of orthogonal Legendre wavelets is attained:

$$
\eta_{n m}(\xi)= \begin{cases}\sqrt{m+\frac{1}{2}} 2^{\frac{k}{2}} L_{m}\left(2^{k} \xi-\hat{n}\right) & \text { for } \frac{\hat{n}-1}{2^{k}} \leq \xi \leq \frac{\hat{n}+1}{2^{k}} \\ 0 & \text { otherwise. }\end{cases}
$$

For $m=0,1, \ldots, M-1$, where $M$ is a positive integer, $\eta_{n m}(\xi)$ forms a basis of $\mathbf{L}^{2}([0,1))$, and the coefficient $\sqrt{m+1 / 2}$ is for orthonormality. Here, $L_{m}\left(2^{k} \xi-\hat{n}\right)$ are the shifted Legendre polynomials of order $m$, which are attained by dilating and translating the well-known Legendre polynomials. These polynomials are constructed with the following recurrence formulae $[24,25]$ :

$$
L_{m}(\tau)=\sum_{k=0}^{m}(-1)^{m+k} \frac{(m+k) !}{(m-k) !(k !)^{2}} \tau .
$$

A function $v(\tau) \in \mathbf{L}^{2}([0,1))$ can be expanded by infinite series of Legendre wavelets as

$$
v(\tau)=\sum_{n=1}^{+\infty} \sum_{m=0}^{+\infty} e_{n m} \eta_{n m}(\tau)
$$

where $e_{n m}=\left\langle\nu(\tau), \eta_{n m}(\tau)\right\rangle$, and $\langle\cdot, \cdot\rangle$ denotes the inner product. If Eq. (15) is truncated, then it can be written as

$$
v(\tau)=\sum_{n=1}^{2^{k-1}} \sum_{m=0}^{M-1} v_{n m} \eta_{n m}(\tau)=V^{T} \Phi(\tau)
$$

where $V^{T}$ and $\Phi(\tau)$ are $2^{k-1} M \times 1$ matrices defined as

$$
V=\left[v_{10}, v_{11}, \ldots, v_{1 M-1}, v_{20}, \ldots, v_{2 M-1}, \ldots, v_{2^{k-1} 0}, \ldots, v_{2^{k-1} M-1}\right]^{T}
$$

and

$$
\begin{aligned}
\Phi(\tau)= & {\left[\eta_{10}(\tau), \eta_{11}(\tau), \ldots, \eta_{1 M-1}(\tau),\right.} \\
& \left.\eta_{20}(\tau), \ldots, \eta_{2 M-1}(\tau), \ldots, \eta_{2^{k-1} 0}(\tau), \ldots, \eta_{2^{k-1} M-1}(\tau)\right]^{T} .
\end{aligned}
$$


Analogously, any arbitrary two-dimensional function $v(x, t)$ on $[0,1) \times[0,1)$ may be expanded by the truncated series of Legendre wavelets as

$$
v(x, t)=\sum_{i=1}^{\hat{m}} \sum_{j=1}^{\hat{m}} \nu_{i j} \phi_{i}(x) \phi_{j}(t)=\Phi^{T}(x) \mathrm{V} \Phi(t),
$$

where $\mathrm{V}=\left[v_{i j}\right]$ and $v_{i j}=\left\langle\phi_{i}(x),\left\langle v(x, t), \phi_{j}(t)\right\rangle\right\rangle$. The operational matrix of fractional integration of Legendre wavelets is defined as

$$
P^{v} \cong \Psi_{\hat{m} \times \hat{m}} F_{B}^{v} \Psi_{\hat{m} \times \hat{m}}^{-1},
$$

where $\Psi_{\hat{m} \times \hat{m}}$ is the matrix of Legendre wavelets, and $P^{v}$ is defined as

$$
F_{B}^{v}=\frac{1}{\hat{m}^{v}} \frac{1}{\Gamma(v+2)}\left(\begin{array}{ccccc}
1 & \delta_{1} & \delta_{2} & \cdots & \delta_{\hat{m}-1} \\
0 & 1 & \delta_{1} & \cdots & \delta_{\hat{m}-2} \\
0 & 0 & 1 & \cdots & \delta_{\hat{m}-3} \\
\vdots & \vdots & 0 & \ddots & \vdots \\
0 & 0 & 0 & 0 & 1
\end{array}\right)
$$

where $\delta_{i}=(i+1)^{\nu+1}-2 i^{\nu+1}+(i-1)^{v+1}[7]$.

Theorem 4.1 Let $\frac{\partial^{3} v(x, t)}{\partial t \partial x^{2}}$ be a continuous and bounded function for $(x, t) \in(0,1) \times(0,1)$, that is, there is $M>0$ such that $\left|\frac{\partial^{3} v(x, t)}{\partial t \partial x^{2}}\right| \leq M$. Then $v(x, t)$ can be expanded as an infinite series of Legendre wavelets, that is,

$$
v(x, t)=\sum_{i=1}^{\infty} \sum_{j=1}^{\infty} c_{i j} \phi_{i}(x) \phi_{j}(t)
$$

where $c_{i j}=\left\langle v(x, t), \phi_{i}(x) \phi_{j}(t)\right\rangle$, and $\langle\cdot, \cdot\rangle$ is the inner product of $v(x, t)$ and $\phi_{i}(x) \phi_{j}(t)$, and this series $v_{m}(x, t)$ converges uniformly to the function $v(x, t)$.

\subsection{The differential evolution}

For the optimization purpose, the differential evolution (DE) algorithm is utilized in this endeavor. This effective heuristic optimizing technique was proposed by Storn and Price [26]. Among many other global optimizers, DE is considered to be more significant for its simplicity and strong population-based stochastic search technique over a continuous domain. The key features of DE are three control parameters, that is, the population size $N P$, crossover constant $C R$, and scaling factor $S f$. These parameters may extensively affect the optimization performance of the DE; therefore, in $[26,27]$ some simple rules are defined for the selection of these parameters. Thus, in the DE algorithm, the solutions are easily obtained by just specifying the population set, an approximate solution, and the objective function. 


\section{Implementation of LWOM}

In this section, we implement the LWOM on the FPDE stated in Eq. (9). Using all the expansions as defined in Section 4, the components of Eq. (9) are discretized as

$$
\frac{\partial^{\gamma_{+}+1} v(x, t)}{\partial t \partial_{+} x^{\gamma}}=\Phi^{T}(x) \mathrm{V} \Phi(t)
$$

where V and $\Phi(\cdot)$ are defined in Eqs. (17) and (18), respectively. Integrating Eq. (22) with respect to $t$, we get

$$
\frac{\partial^{\gamma_{+}} v(x, t)}{\partial_{+} x^{\gamma}}=\Phi^{T}(x) \mathrm{V} P_{t}^{1} \Phi(t)+\frac{\partial^{\gamma_{+}} s(x)}{\partial_{+} x^{\gamma}} .
$$

Now, by fractional integration of order $\gamma$ of Eq. (23) with respect to $x$ from $L$ to $x$ we get

$$
v(x, t)=\left(P_{x}^{\gamma} \Phi(x)\right)^{T} \mathrm{~V} P_{t}^{1} \Phi(t)+I_{+}^{\gamma}(s(x))-(x-L) s(L)+\left.(x-L) \frac{\partial^{\gamma_{+}}}{\partial_{+} x^{\gamma}}(v(x, t))\right|_{x=L},
$$

where $I_{+}^{\gamma}(s(x))$ is the right Riemann-Liouville fractional integral of $s(x)$. Let $x=R$. Then

$$
\left.\frac{\partial^{\gamma_{+}}}{\partial_{+} x^{\gamma}}(v(x, t))\right|_{x=L}=\frac{1}{(R-L)}\left(b(R, t)-\left(P_{x}^{\gamma} \Phi(R)\right)^{T} \mathrm{~V} P_{t}^{1} \Phi(t)-I_{+}^{\gamma}(s(R))+(R-L) s(L)\right) .
$$

Substituting Eq. (25) into Eq. (24) and Eq. (24) into Eq. (23), we get the approximation for $v(x, t)$ :

$$
\begin{aligned}
v(x, t)= & -\left(P_{x}^{\gamma} \Phi(x)\right)^{T} \mathrm{~V} P_{t}^{1} \Phi(t)+I_{-}^{\gamma} s(x)-(R-x) s(L) \\
& -\left(R-\left(\frac{x^{\gamma+1}}{\Gamma(\gamma+2)}-\frac{L^{\gamma+1}}{\Gamma(\gamma+2)}\right)\right) \frac{\partial^{\gamma+} s(L)}{\partial_{+} x^{\gamma}} \\
& +\left(R-\left(\frac{x^{\gamma+1}}{\Gamma(\gamma+2)}-\frac{L^{\gamma+1}}{\Gamma(\gamma+2)}\right)\right) \mathrm{H}(R, L, t),
\end{aligned}
$$

where

$$
\mathrm{H}(R, L, t)=\frac{1}{(R-L)}\left(\left(P_{x}^{\gamma} \Phi(L)\right)^{T} \mathrm{~V} P_{t}^{1} \Phi(t)-I_{-}^{\gamma}\left(s^{\gamma_{+}}(L)\right)+(R-L) \frac{\partial^{\gamma_{+}} s(R)}{\partial_{+} x^{\gamma}}\right) .
$$

Differentiating Eq. (26) with respect to $t$, we get

$$
\begin{aligned}
\frac{\partial v(x, t)}{\partial t}= & -\left(P_{2}^{\gamma} \Phi(x)\right)^{T} \mathrm{~V} \Phi(t)+I_{-}^{\gamma} s(x)-(R-x) s(L) \\
& -\left(R-\left(\frac{x^{\gamma+1}}{\Gamma(\gamma+2)}-\frac{L^{\gamma+1}}{\Gamma(\gamma+2)}\right)\right) \frac{\partial^{\gamma+} s(L)}{\partial_{+} x^{\gamma}} \\
& +\left(R-\left(\frac{x^{\gamma+1}}{\Gamma(\gamma+2)}-\frac{L^{\gamma+1}}{\Gamma(\gamma+2)}\right)\right) \frac{\partial \mathrm{H}(R, L, t)}{\partial t} .
\end{aligned}
$$

Now, substituting Eqs. (23) and (26)-(27) into Eq. (9), we achieve the residual function (fitness function) as

$$
\boldsymbol{R}\left(v_{i j}\right)=\frac{1}{\hat{m}^{2}} \sum_{i=1}^{\hat{m}} \sum_{j=1}^{\hat{m}}\left(x_{i}, t_{j}, P_{x}^{\gamma}, P_{t}^{1}, \Phi\left(x_{i}\right), \Phi\left(t_{j}\right)\right)^{2} .
$$




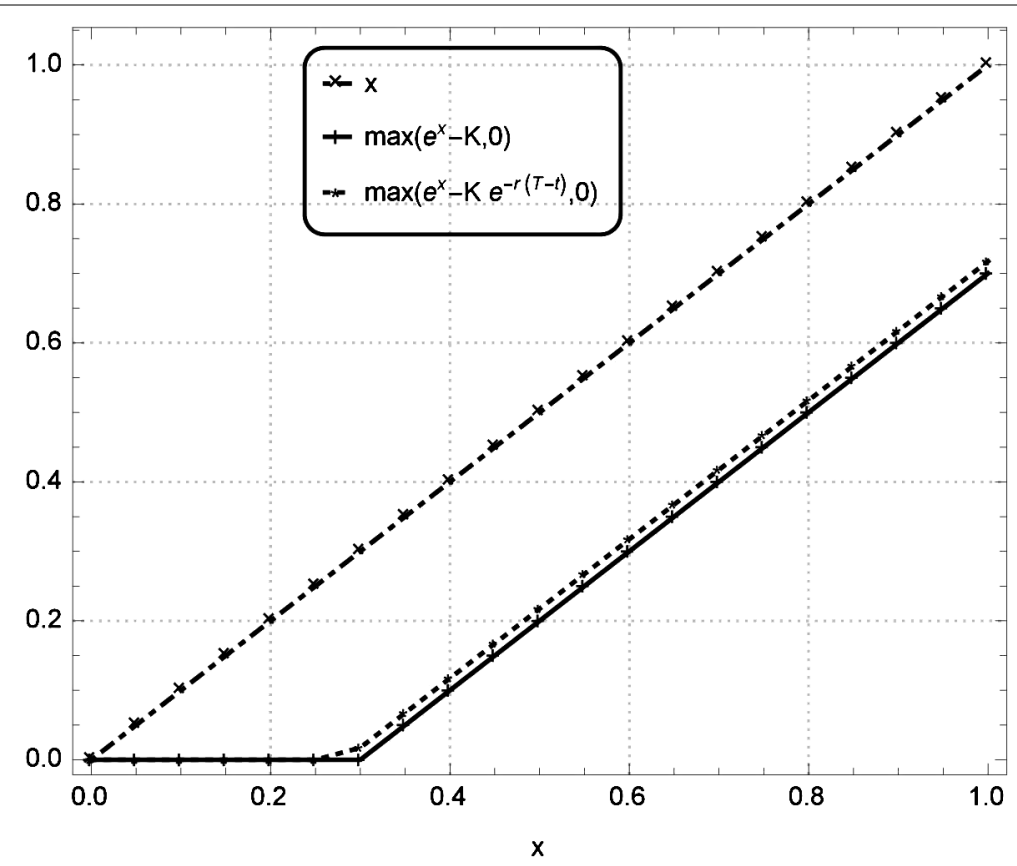

(a) $K=0.3$

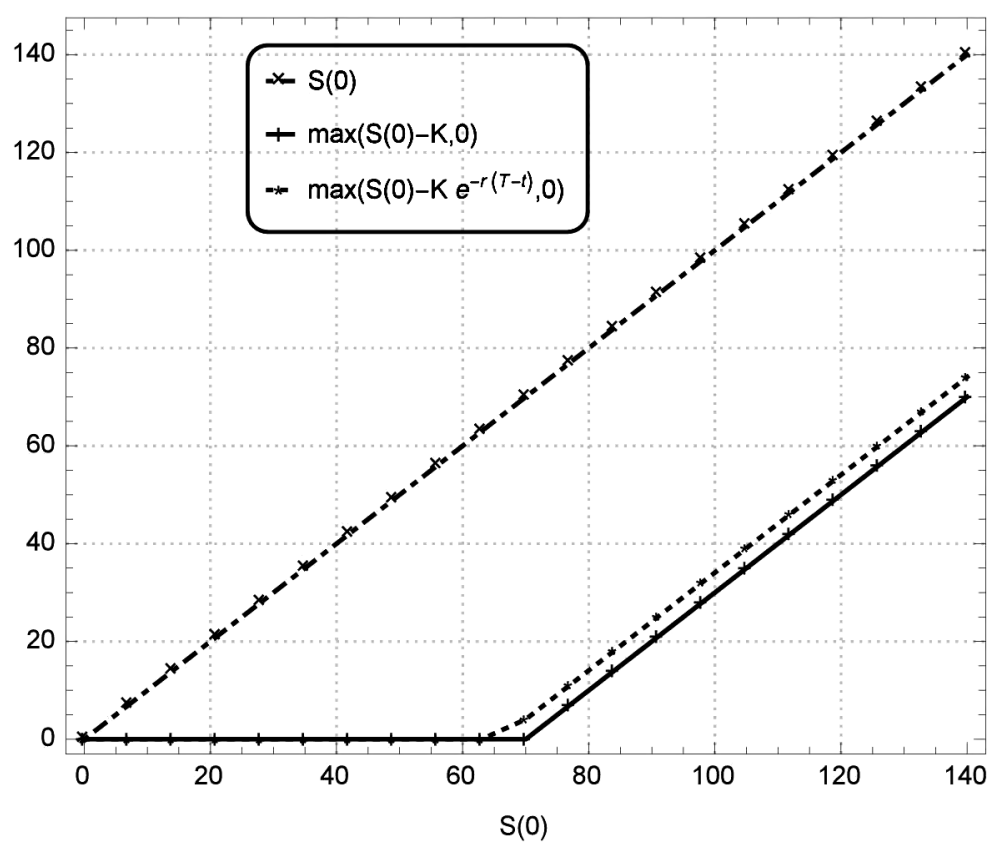

(b) $K=70$

Figure 1 Price values of underlying asset.

Following the differential evolution algorithm, we obtain the optimized values of the unknown parameters $v_{i j}$ for $i, j=1,2, \ldots, \hat{m}$. Replacing these values in Eq. (26), the approximate solution of $v(x, t)$ is acquired. All the numerical manipulations of DE are carried out using Mathematica 10. Consequently, the graphical solutions of $v(x, t)$ for different values of stock market parameters, such as interest rate, maturity, time, etc., are plotted in Figures 1, 2, 3, 4. 


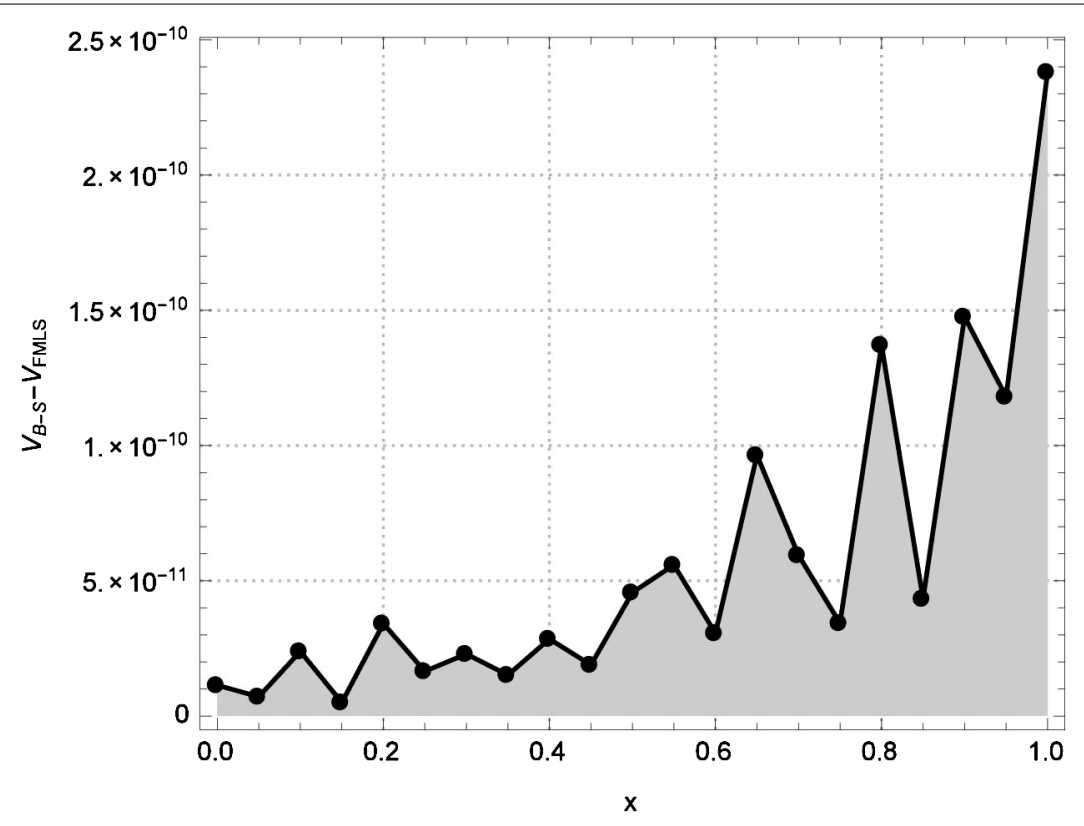

(a) $\gamma=2$ and $\sigma=0.1$

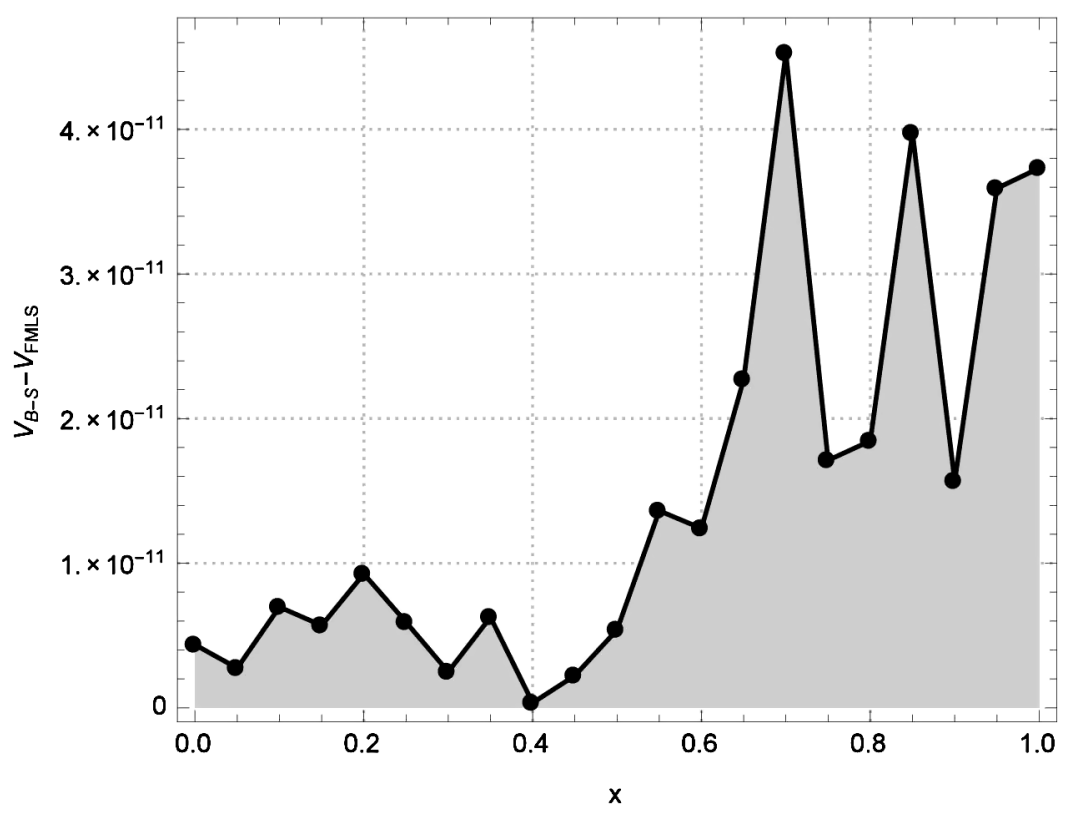

(b) $\gamma=1.2$ and $\sigma=0.01$

Figure 2 Comparison of BS versus FMLS solutions at $t=1$.

The investigations analyze the behavior of the fractional diffusion equation in the field of finance, exclusively in pricing the derivatives. Productively, graphical results at various values of parameters and with different orders of derivatives are depicted. Figures 1(a)-(b) show the asset prices of the model in the specified domain. The high convergence of the proposed method from the FMLS to BS models for special values of $\gamma$ and $\sigma$ is presented in Figures 2(a)-(b). In addition, simulated stock paths of the BS and FMLS models can be seen in Figure 3. The European call options of both the models converge as $\gamma \rightarrow 2$, which is 


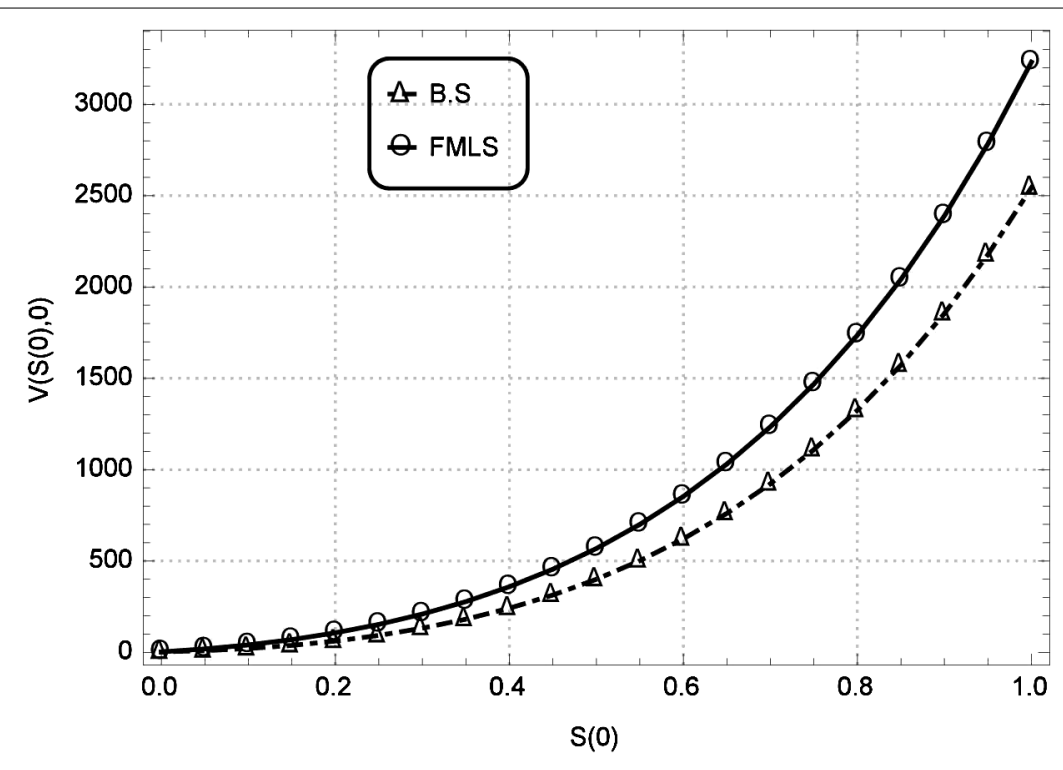

Figure 3 Simulated stock paths of BS and FMLS models at $\gamma=2, t=0$.

exhibited in Figures 4(a)-(b). The minimum values of the residual function $\boldsymbol{R}\left(v_{i j}\right) \leq 10^{-k}$ for positive integers $k=1,2, \ldots, 15$ can be seen in Table 1 . The comparison between LWOM and q-HATM [22] is presented in Tables 2 and 3. Generally, LWOM solutions match with the q-HATM up to two to three decimal places.

\section{Conclusions}

This paper embodied the exploration of some applications of fractional partial differential equations in financial mathematics. Since the solutions of financial models are advantageous to illustrate multiple behaviors of the stock market prices, we undertook some wellknown financial models, namely, the Black-Scholes (BS) model and a class of Lévy models, finite moment log stable model (FMLS). A new amalgamation of two eminent techniques, Legendre wavelets and differential evolution, was introduced for numerical demonstration of stock market parameters of these models. Thus, from the facts and figures it is possible to conclude that:

- The Legendre wavelets optimization method is successfully implemented in the fractional partial differential models that occur in financial modeling.

- Legendre wavelets produce a good approximation of arbitrary functions.

- LWOM enables to interpret the effects of the parameters smoothly.

- The numerical solutions of the model enable us to locate the pricing variations and the parameters affecting the stock market.

- From the simulated stock paths of the BS and FMLS models it can be easily depicted that the European call options of both the models converge as $\gamma \rightarrow 2$.

- Since Lévy models consider the jumps of the market, FMLS being a class of Lévy models shows an incremental path of the stock prices at $t=0$, which differs from the path of the BS model.

- By comparison of the tables the solutions obtained from LWOM are found to be in a good agreement with q-HATM at particular $\hbar$. 


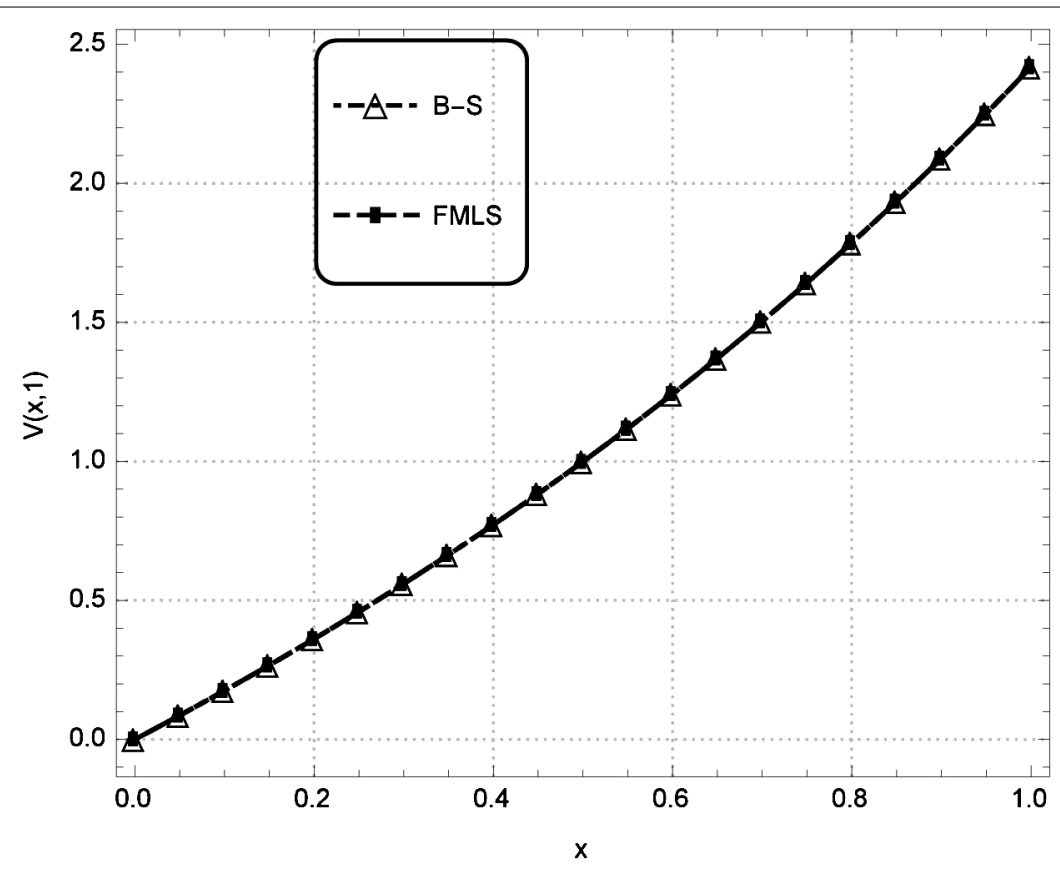

(a)

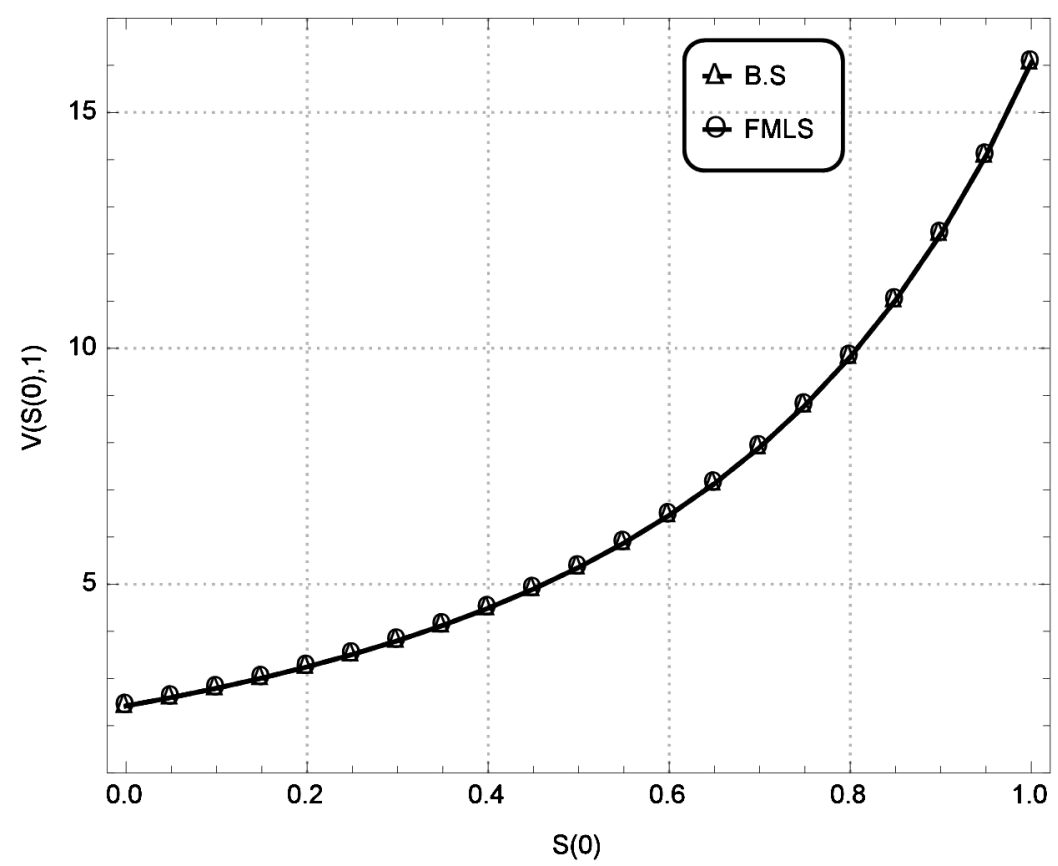

(b)

Figure 4 European call options of BS and FMLS models at $\gamma=2, t=1$.

- Since efficiency of LWOM does not depend on any auxiliary parameter as that of q-HATM, it converges toward an accurate approximation more competently with less time consumption. 
Table 1 Global optimum residual errors $R\left(v_{i j}\right)$ for $N P=20$

\begin{tabular}{|c|c|c|c|c|}
\hline \multirow[t]{2}{*}{$\gamma$} & \multicolumn{2}{|l|}{ BS } & \multicolumn{2}{|l|}{ FMLS } \\
\hline & $\sigma=0.01$ & $\sigma=0.1$ & $\sigma=0.01$ & $\sigma=0.1$ \\
\hline 2.0 & $9.4463 \times 10^{-8}$ & $7.3315 \times 10^{-15}$ & $2.0887 \times 10^{-15}$ & $7.3314 \times 10^{-15}$ \\
\hline 1.2 & $8.7099 \times 10^{-15}$ & $7.3312 \times 10^{-15}$ & $6.9499 \times 10^{-15}$ & $4.2934 \times 10^{-15}$ \\
\hline
\end{tabular}

Table 2 Comparison of LWOM with q-HATM [22] at $\sigma=0.1, x=1$, and $n=3$

\begin{tabular}{|c|c|c|}
\hline \multirow[t]{2}{*}{$t$} & \multicolumn{2}{|l|}{$\alpha=2$} \\
\hline & $\begin{array}{l}\text { q-HATM } \\
\hbar=\mathbf{0 . 0 0 0 8 5}\end{array}$ & LWOM \\
\hline 0.2 & 0.383141 & 0.361403 \\
\hline 0.4 & 0.759879 & 0.771825 \\
\hline 0.6 & 1.223750 & 1.242120 \\
\hline 0.8 & 1.796510 & 1.785540 \\
\hline 1.0 & 2.475530 & 2.418280 \\
\hline
\end{tabular}

Table 3 Comparison of LWOM with q-HATM [22] at $\sigma=0.1, t=1$, and $n=3$

\begin{tabular}{lll}
\hline $\boldsymbol{x}$ & $\boldsymbol{\alpha}=\mathbf{2}$ & \\
\cline { 2 - 3 } & $\begin{array}{l}\mathbf{q}-\text { HATM } \\
\hbar=-\mathbf{0 . 0 0 0 8 3}\end{array}$ & LWOM \\
\hline 0.2 & 2.432330 & 2.432340 \\
0.4 & 2.428880 & 2.428890 \\
0.6 & 2.425380 & 2.425400 \\
0.8 & 2.421850 & 2.421860 \\
1.0 & 2.418270 & 2.418280 \\
\hline
\end{tabular}

- The proposed technique is appropriate for different financial models that can be expressed as partial or ordinary differential equations of integer and fractional orders, subjected to initial/boundary conditions.

Thus, this paper contributes a new method of finding solutions of FPDEs and presents wide applications of FPDEs in management sciences.

\section{Acknowledgements}

Author Najeeb Alam Khan would like to thank the Dean faculty of science of the University of Karachi for supporting this research.

\section{Competing interests}

The authors declare that they have no competing interests.

Authors' contributions

All authors contributed equally to this work. All authors read and approved the final manuscript.

\section{Author details}

${ }^{1}$ Department of Computer Science, Muhammad Ali Jinnah University, Karachi, 75400, Pakistan. ${ }^{2}$ Department of Mathematics, University of Karachi, Karachi, 75270, Pakistan. ${ }^{3}$ Department of Humanities \& Natural Sciences, Bahria University, Karachi, 75260, Pakistan. ${ }^{4}$ Department of Electrical Engineering, COMSATS Institute of Information Technology, Attock, Pakistan.

\section{Publisher's Note}

Springer Nature remains neutral with regard to jurisdictional claims in published maps and institutional affiliations.

Received: 14 September 2017 Accepted: 28 December 2017 Published online: 10 January 2018

\section{References}

1. Merton, RC: Option pricing when underlying stock returns are discontinuous. J. Financ. Econ. 3, 125-144 (1976)

2. Bates, D: Jump and stochastic volatility: exchange rate processes implicit in Deutsche mark in options. Rev. Financ. Stud. 9, 69-107 (1996) 
3. Heston, SL: A closed-form solution for options with stochastic volatility with applications to bond and currency options. Rev. Financ. Stud. 6(2), 327-343 (1993)

4. Singh, VK: Pricing competitiveness of jump-diffusion option pricing models: evidence from recent financial upheavals. Stud. Econ. Finance 32(3), 357-378 (2015)

5. Cartea, A, del-Castillo-Negrete, D: Fractional diffusion models of option prices in markets with jumps. Physica A 374 , 749-763 (2007)

6. Company, R, Jódar, L, Pintos, JR: A numerical method for European option pricing with transaction costs nonlinear equation. Math. Comput. Model. 50, 910-920 (2009)

7. Robinson, GK: Practical computing for finite moment log-stable distributions to model financial risk. Stat. Comput. 25(6), 1233-1246 (2015)

8. Carr, $\mathrm{P}, \mathrm{Wu}, \mathrm{L}$ : The finite moment log stable process and option pricing. J. Finance 58(2), 753-777 (2003)

9. Carr, P, Geman, H, Madan, D, Yor, M: The fine structure of asset returns: an empirical investigation. J. Bus. 75(2), 305-332 (2002)

10. Cartea, A, del Castillo-Negrete, D: Fractional diffusion models of option prices in markets with jumps. Physica A 374 , 749-763 (2007)

11. Koponen, I: Analytic approach to the problem of convergence of truncated Levy flights towards the Gaussian stochastic process. Phys. Rev. E 52(1), 1197-1199 (1995)

12. Boyarchenko, SI, Levendorskii, SZ: Non-Gaussian Merton-Black-Scholes Theory. World Scientific, Singapore (2002)

13. Khan, NA, Razzaq, OA, Ayaz, M: Some properties and applications of conformable fractional Laplace transform (CFLT). J. Fract. Calc. Appl. 9(1), 72-81 (2018)

14. Caputo, M, Fabrizio, M: A new definition of fractional derivative without singular kernel. Prog. Fract. Differ. Appl. 1(2), 73-85 (2015)

15. Atangana, A, Baleanu, D: New fractional derivatives with non-local and non-singular kernel theory and application to heat transfer model. Therm. Sci. 20(2), 763-769 (2016)

16. Singh, J, Kumar, D, Qurashi, MA, Baleanu, D: A new fractional model for giving up smoking dynamics. Adv. Differ. Equ. 2017, Article ID 88 (2017)

17. Singh, J, Kumar, D, Baleanu, D: On the analysis of chemical kinetics system pertaining to a fractional derivative with Mittag-Leffler type kernel. Chaos 27, 103-113 (2017)

18. Marom, $\mathrm{O}$, Momoniat, $\mathrm{E}$ : A comparison of numerical solutions of fractional diffusion models in finance. Nonlinear Anal., Real World Appl. 10, 3435-3442 (2009)

19. Delgado, VFM, Aguilar, JFG, Martinez, YH, Baleanu, D, Jimenez, RFE, Peregrino, VHO: Laplace homotopy analysis method for solving linear partial differential equations using a fractional derivative with and without kernel singular. Adv. Differ. Equ. 2016, Article ID 164 (2016)

20. Acan, O, Baleanu, D: A new numerical technique for solving fractional partial differential equations (2017). arXiv: 1704.02575

21. Ziane, D, Baleanu, D, Belghaba, K, Cheirf, MH: Local fractional Sumudu decomposition method for linear partial differential equations with local fractional derivative. J. King Saud Univ., Sci. (2017). https://doi.org/10.1016/j.jksus.2017.05.002

22. Srivastava, HM, Kumar, D, Singh, J: An efficient analytical technique for fractional model of vibration equation. Appl. Math. Model. 45, 192-204 (2017)

23. Singh, J, Kumar, D, Nieto, JJ: A reliable algorithm for local fractional Tricomi equation arising in fractal transonic flow. Entropy 18, Article ID 206 (2016)

24. Heydari, MH, Hooshmandasl, MR, Mohammadi, F: Legendre wavelets method for solving fractional partial differential equations with Dirichlet boundary conditions. Appl. Math. Comput. 234, 267-276 (2014)

25. Khan, NA, Razzaq, OA: An efficient computer based wavelets approximation method to solve fuzzy boundary value differential equations. Nonlinear Eng. 5(1), 1-6 (2016)

26. Storn, R, Price, K, Lampinen, JA: Differential Evolution: A Practical Approach to Global Optimization. Springer, Berlin (2005)

27. Saruhan, H: Differential evolution and simulated annealing algorithms for mechanical systems design. Int. J. Eng. Sci. Technol. 17, 131-136 (2014)

28. Meerschaert, MM, Tadjeran, C: Finite difference approximations for two-sided space-fractional partial differential equations. Appl. Numer. Math. 56(1), 80-90 (2006)

29. Mao, Z, Liang, Z, Lian, J, Zhang, H: Evaluation of the stochastic modelling on options. Int. J. Eng. Res. Appl. 2(3)، 2463-2473 (2012)

\section{Submit your manuscript to a SpringerOpen ${ }^{\circ}$ journal and benefit from:}

- Convenient online submission

- Rigorous peer review

- Open access: articles freely available online

- High visibility within the field

- Retaining the copyright to your article

Submit your next manuscript at $\gg$ springeropen.com 